\title{
A CFD analysis of the performance characteristics of different Darrieus turbine runners
}

\author{
Rossen Iliev ${ }^{1, *}$ \\ ${ }^{1}$ Tehnical University of Sofia, Department of Hydroaerodynamics and Hydraulic Machines, Bulgaria
}

\begin{abstract}
This paper presents the capabilities of analyzing different Darrieus wind turbine runners with the computer program Ansys Fluent. A $\mathrm{K}$-omega turbulence model was used in the case of a two-dimensional flow with a suitable computational grid around the profile of the blades. The obtained theoretical performance characteristics were validated on test rig №7 (Wind Turbines) in the Laboratory of Hydropower and Hydraulic Turbomachinery (HEHT Lab) at the Technical University of Sofia. The data analysis shows that it's possible to predict the performance characteristic and the optimum operating regime of the Darrieus wind turbine.
\end{abstract}

\section{Introduction}

The most commonly used mathematical models for calculation of tangential forces acting on wind turbine blades consider the runner as an 'active disk' [1, 2]. It is assumed to be an imaginary rotating body covered by one or more stream tubes. The induced velocities through the 'disk' are considered to be constant. The calculations of the acting forces are performed for a rotating blade that crosses the stream tubes at a given moment. The chord of this blade has a length equal to the sum of the chord lengths of all the blades in the real runner. This simplified scheme is insufficient for a more in-depth study of the workflow. It gives satisfactory results in some cases with two and three-bladed runners (depends on solidity).

The complex unsteady flow in most cases is impossible to be studied with classical stream tube models. Therefore, we go to the so-called numerical modelling of flows - Computational Fluid Dynamics (CFD). CFD modelling gives us detailed information about the flow (temperature, pressure, velocity field, etc.) at each point of the computational space. It calculates the stresses on streamlined surfaces and gives us the opportunity to visualize these results in the form of colour contours, isolines, graphs and stream line pictures. The results obtained by the CFD modelling can be compared with the results obtained by experimental research, which significantly reduces the time for conducting physical experiments. A sequential application of a numerical and physical experiment gives us the opportunity to analyse the flow through a synthesized blade system of a wind turbine runner.

\footnotetext{
* Corresponding author: riliev@tu-sofia.bg
} 


\section{Creating a CFD numerical model}

\subsection{Geometric dimensions and boundary conditions}

The studied wind turbine has working blades with a constant cross section, which allows us to switch from a three-dimensional to a two-dimensional numerical model. This significantly reduces the computation time and resources used by the program, which eliminates the need for expensive computers (clusters).

Fig. 1 show a 3D model and a section view of the turbine runner. The blades -2 are a part of a cylindrical surface with a radius of curvature $\mathrm{R}=100 \mathrm{~mm}$, placed on a cylindrical surface with a diameter $\mathrm{D}_{0}=800 \mathrm{~mm}$ [3] with a pitch angle $\varphi_{\mathrm{r}}$. They are attached to two disks -3 , which are mounted on a fixed axis $-1 . D_{1}$ defines the outer diameter of the runner. It is used in the calculation of the power factor $-c_{p}$ and the speed ratio TSR of the wind turbine $[4,5]$.

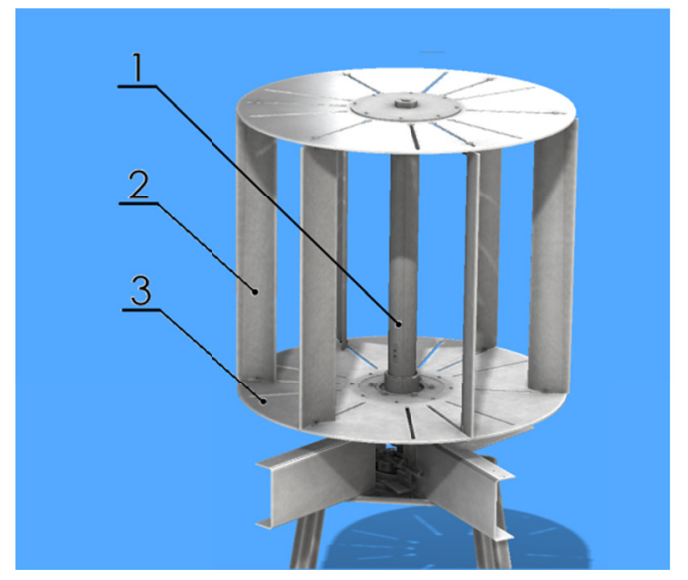

a.

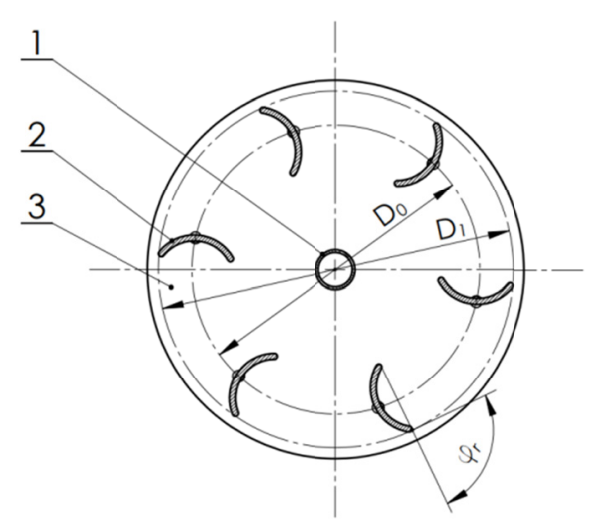

b.

Fig. 1. Turbine runner

In order to create an adequate two-dimensional numerical model of a rotating wind turbine, the 'Sliding mesh' technique is most commonly used in practice. It is expressed in the exchange of data between two separate areas, the points of contact of which form a closed loop. The first area is stationary while the other rotates at a set angular velocity, sliding along the closed loop. Fig. 2 shows a schematic view of the used computational domains and their boundary conditions. The largest quadratic area is the surrounding area in which the wind turbine is positioned, the large circular area is the runner and the smaller - the fixed axis.

The inlet, velocity and turbulence of the flow are defined by the boundary condition 'Velocity inlet'. 'Pressure-outlet' sets the static pressure at the outlet of the domain (in this case it is $1 \mathrm{~atm})$. The 'interface-1' contour performs the above-mentioned flow data exchange between the surrounding area and the runner area, while 'interface-2' performs a data exchange between the turbine runner and the fixed axis areas. The upper and lower sides of the surrounding space are defined as walls that do not take into account viscous forces (free slip wall). The contours of the blades and the fixed axis are set as perfectly smooth walls ('Wall').

In some publications [6-8] it is mentioned that the size of the surrounding space has a direct impact on the final results of the simulation. Table 1 presents the sizes of the 
recommended domains. The dimensions $\mathrm{W}, \mathrm{L}_{1}$ and $\mathrm{L}_{2}$ have been increased 3 times so any influence on the side walls is avoided.

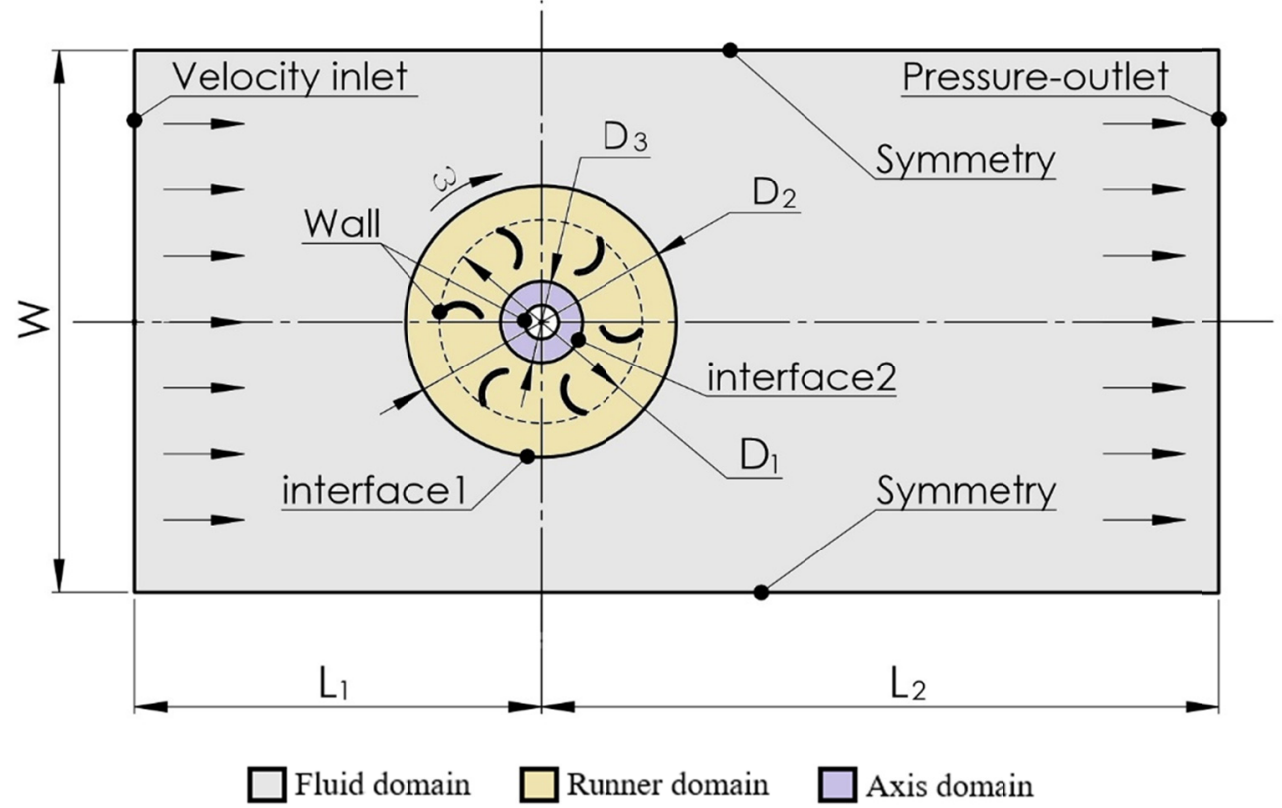

Fig. 2. Dimensions and boundary conditions of the computational domains.

Table 1. Recommended geometric dimensions of the domains.

\begin{tabular}{|c|c|c|c|}
\hline $\mathrm{D}_{2}$ & $\mathbf{L}_{1}$ & $\mathrm{~L}_{2}$ & $\mathrm{~W}$ \\
\hline $1.5 . \mathrm{D}_{1}$ & $10 . \mathrm{D}_{1}$ & $20 . \mathrm{D}_{1}$ & $10 . \mathrm{D}_{1}$ \\
\hline
\end{tabular}

\subsection{A CFD grid}

Apart from the geometry of the model, the accuracy of the solution depends on the correct modelling of the laminar boundary layer on the surface of the blade. For this reason, the coefficient $\mathrm{y}^{+}[6-8]$ has been introduced, which measures the density of the mesh near the blade wall.

$$
y^{+}=\frac{\rho \cdot U_{t} \cdot y}{\mu}
$$

In the equation above $\rho$ is the fluid density, $U_{t}-$ the tangential (frictional) velocity along the streamlined surface, $y$ - the height of the first layer of cells along the streamlined contour (measured at the normals of the surface) and $\mu$ - the dynamic viscosity of the fluid.

A lot of calculators can be found on the Internet which calculate the required height ' $y$ ' by setting the background flow velocity $-\mathrm{c}_{\mathrm{w}}$, the chosen coefficient $\mathrm{y}+$, the dynamic viscosity of the fluid and the reference length of the streamlined body (in this case the chord of the airfoil) - L. After generating the grid, it is necessary to check the coefficient $\mathrm{y}^{+}$ by running a test calculation. If all values of $\mathrm{y}^{+}$along the contour of the wall are equal or below the needed one, the condition is met.

Fig. 3 shows pictures of the generated CFD grids of the three domains. Table 2 shows the Ansys-recommended limits for CFD grid parameters. Table 3 presents the dimensions 
of the computational rectangular structured mesh in the area of the boundary layer of the turbine blades. It has a growth factor of 1.05 , a maximum cell size

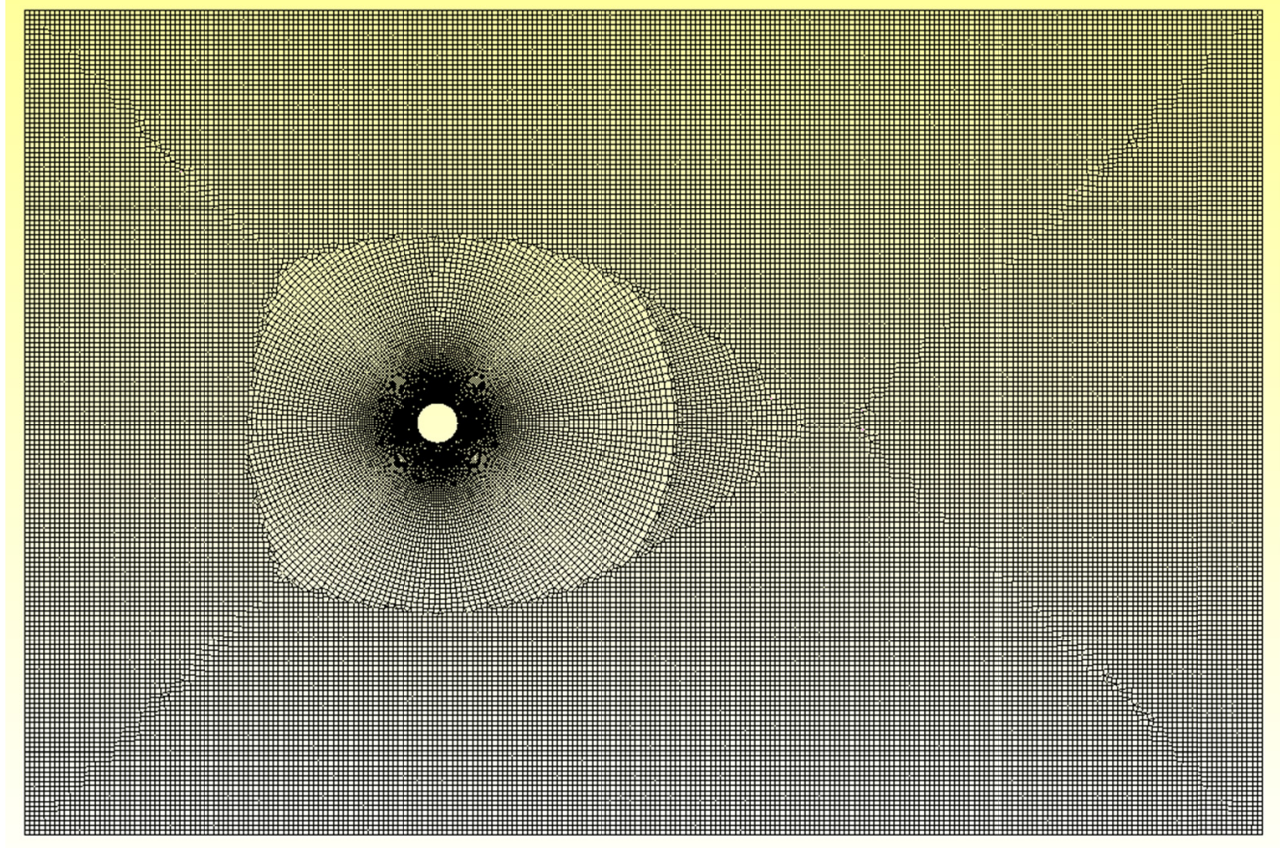

a.

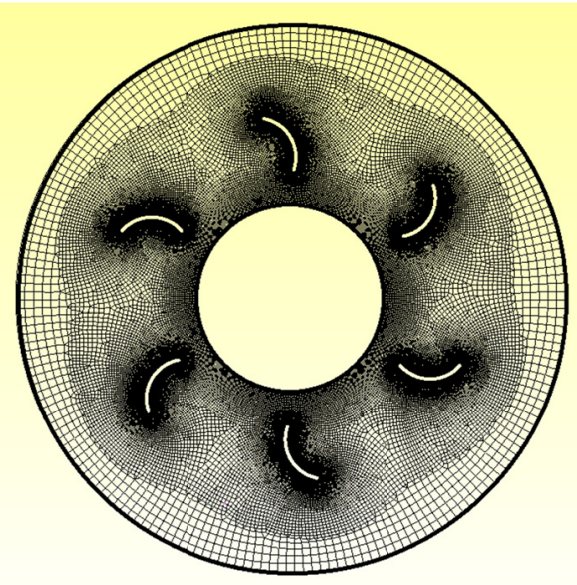

b.

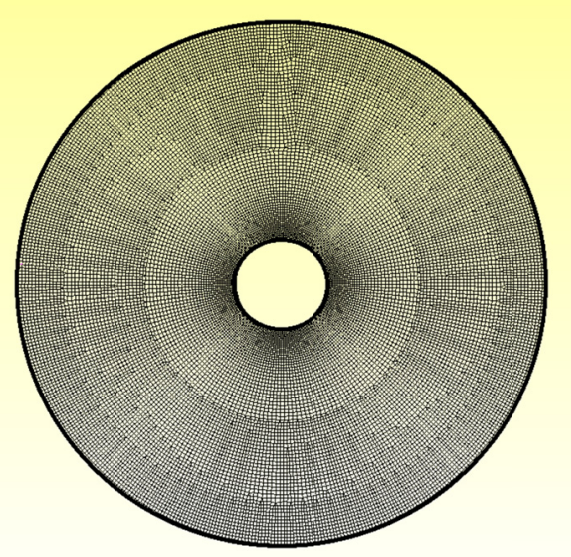

c.

Fig. 3. Computational grid of the domains ( $\mathrm{a}$ - Fluid domain, $\mathrm{b}$ - Runner domain, $\mathrm{c}-$ Axis domain).

ratio (AR) of 22.5 and a minimum orthogonal quality of 0.86 . These parameters are much over the recommended minimum, which guarantees the grid quality. The values of the grid parameters of the three domains are shown in table 4. In order to maintain a sufficiently low aspect ratio between adjacent cells, a thickening ratio of 0.99 was used. 
Table 2. Recommended CFD grid parameters

\begin{tabular}{|c|c|c|}
\hline $\begin{array}{c}\text { Max. volume } \\
\text { ratio }\end{array}$ & Min. orthogonal quality & Max.aspect ratio \\
\hline 15 & 0.2 & 60 \\
\hline
\end{tabular}

Table 3. Boundary layer grid parameters of the turbine blades.

\begin{tabular}{|c|c|c|c|c|c|c|}
\hline $\begin{array}{l}\text { Max. cell } \\
\text { size }\end{array}$ & Min. cell size & Wall distance & Min. orth. quality & Max. AR & Layers & $\begin{array}{l}\text { Growth } \\
\text { rate }\end{array}$ \\
\hline $1 \mathrm{~mm}$ & $0.180 \mu \mathrm{m}$ & $8 \mu \mathrm{m}$ & 0.86 & 22.5 & 30 & 1.05 \\
\hline
\end{tabular}

Table 4. Grid parameters of the computational domains.

\begin{tabular}{|c|c|c|c|c|}
\hline \multicolumn{5}{|c|}{ Fluid domain } \\
\hline $\begin{array}{l}\text { Max. cell } \\
\text { size }\end{array}$ & Min. cell size & Min. orthogonal quality & Max.aspect ratio & Number of cells \\
\hline $245 \mathrm{~mm}$ & $22 \mathrm{~mm}$ & 0.85 & 2.1 & 160506 \\
\hline \multicolumn{5}{|c|}{ Runner domain } \\
\hline $\begin{array}{l}\text { Max. cell } \\
\text { size }\end{array}$ & Min. cell size & Min. orthogonal quality & Max.aspect ratio & Number of cells \\
\hline $22 \mathrm{~mm}$ & $1 \mathrm{~mm}$ & 0.55 & 2.1 & 4319227 \\
\hline \multicolumn{5}{|c|}{ Axis domain } \\
\hline $\begin{array}{l}\text { Max. cell } \\
\text { size }\end{array}$ & Min. cell size & Min. orthogonal quality & Max.aspect ratio & Number of cells \\
\hline $7 \mathrm{~mm}$ & $1 \mathrm{~mm}$ & 0.81 & 1.756 & 9302 \\
\hline
\end{tabular}

\subsection{The turbulence model}

The turbulence model k- $\omega$ SST (Shear Stress Transport) is a hybrid. It combining the Wilcox k-omega and the k-epsilon models. A blending function activates the Wilcox model near the walls and the k-epsilon model in the free stream. This ensures that the appropriate model is utilized throughout the flow field. The transport equations of the k- $\omega$ model are described below.

Specific dissipation is defined as:

$$
\omega=\frac{\varepsilon}{k}
$$

In equation (2) $\mathrm{k}$ is the turbulence kinetic energy and $\varepsilon$ is the rate of dissipation of turbulence kinetic energy.

The equation for the balance of turbulence kinetic energy $\mathrm{k}[8]$ is:

$$
\frac{\delta(p \cdot k)}{\delta(t)}+\frac{\delta\left(\rho \cdot u_{j} \cdot k\right)}{\delta x_{j}}=\mathrm{P}-\beta . \rho . \omega+\frac{\delta}{\delta x_{j}} \cdot\left\{\left[\mu_{1}+\sigma_{k} \cdot \mu_{t}\right] \cdot \frac{\delta k}{x_{j}}\right\}
$$

where $\beta$ and $\sigma_{\mathrm{k}}$ are constants, $\omega$ is the specific dissipation, $\mu_{1}$ is the molecular viscosity and $\mu_{\mathrm{t}}$ is the turbulence viscosity. 
The first term on the left side of the equation is local, transient, taking into account the degree of change of the turbulence kinetic energy $\mathrm{k}$; the second is convective, taking into account the transfer of $\mathrm{k}$ by convection. The first member on the right of the equation is a source taking into account the degree of generation of $\mathrm{k}$; the second is dissipative, taking into account the degree of dissipation of $\mathrm{k}$, the third is diffuse, taking into account the transfer of $\mathrm{k}$ by diffusion.

The equation of the specific dissipation $\omega[8]$ is:

$$
\frac{\delta(p \cdot \omega)}{\delta(t)}+\frac{\delta\left(\rho \cdot u_{j} \cdot \omega\right)}{\delta x_{j}}=\frac{\gamma}{v_{t}}-\beta \cdot \rho \cdot \omega^{2}+\frac{\delta}{\delta x_{j}} \cdot\left\{\left[\mu_{1}+\sigma_{\omega} \cdot \mu_{t}\right] \cdot \frac{\delta \omega}{x_{j}}\right\}+2 .\left\{1-\mathrm{F}_{1}\right\} \cdot \frac{\rho \cdot \sigma_{\omega}}{\omega} \cdot \frac{\delta k}{\delta x_{j}} \cdot \frac{\delta \omega}{x_{j}}
$$

The first term on the left side of the equation is local, transient, taking into account the degree of change of $\omega$; the second is convective, taking into account the transfer of $\omega$ by convection. The first term on the right-hand side of the equation is the source, taking into account the degree of generation of $\omega$; the second is dissipative, taking into account the degree of dissipation of $\omega$, the third is diffuse, taking into account the transfer of $\omega$ by diffusion; the fourth is 'mixed-diffuse', which is an additional source member, responsible for modelling the transition from $\varepsilon$ to $\omega$.

\section{Compared quantities}

The power factor values are determined by the dependence $[3,8]$ :

$$
\begin{gathered}
c_{p}=\frac{P}{P_{w}} \\
P=M_{b} \cdot \omega=M_{b} \cdot \frac{\pi \cdot n}{30} \cdot T \\
P_{w}=\rho \cdot S \cdot \frac{C_{w, S}^{3}}{2}
\end{gathered}
$$

In equation (5) $\mathrm{P}$ is the effective power (on the shaft) of the turbine and $\mathrm{P}_{\mathrm{w}}$ is the power of the airflow. In equations (6) and (7) $\mathrm{M}_{\mathrm{b}}$ is the torque; $\mathrm{n}-$ angular velocity, $\mathrm{c}_{\mathrm{w}, \mathrm{s}}-$ average wind speed, $\mathrm{S}=\mathrm{H} . \mathrm{D}_{1}$ - cross-section of the runner, perpendicular to the vector of the wind velocity. It should be noted that in the two-dimensional CFD model the dimension $\mathrm{D}_{1}$ is used in equation (7) instead of S. The calculations and experiments were conducted for the same average wind velocity: $\mathrm{c}_{\mathrm{w}, \mathrm{s}}=8.1 \mathrm{~m} / \mathrm{s}$.

For each experiment the so-called speed ratio TSR $[3,8]$ was used. This is the ratio between the tangential speed of the tip of the blades $-\mathrm{u}$ and actual speed of the wind $-\mathrm{c}_{\mathrm{w}, \mathrm{s}}$.

$$
T S R=\frac{u}{c_{w}}
$$

The experiments were performed on test rig №7C in the laboratory of Hydropower and Hydraulic Turbomachinery at the Technical University of Sofia - HEHT [3, 4, 5, 9].

\subsection{Solver settings}

Since the Mach number in the current conditions is below 0.3 we consider the flow to be incompressible. That's why we switch to a pressure-based solver.

This solver allows us to resolve a flow problem in either a segregated or a coupled manner. Ansys Fluent provides the option to choose among five pressure-velocity coupling algorithms: SIMPLE, SIMPLEC, PISO, Coupled, and Fractional Step (FSM) (for unsteady 
flows using the non-iterative time advancement scheme (NITA)). All the aforementioned schemes, except for the 'coupled' scheme, are based on the predictor-corrector approach.

A coupled calculation scheme was used with settings shown in table 5.

Table 5. Spatial discretization settings on a coupled calculation scheme.

\begin{tabular}{|c|c|}
\hline Gradient & Last Square Cell based \\
\hline Pressure & Second Order \\
\hline Momentum & Second Order Upwind \\
\hline Turbulence Kinetic Energy & Second Order \\
\hline Dissipation Rate & Second Order Upwind \\
\hline Transient Formulation & Second Order Implicit \\
\hline
\end{tabular}

The chosen value of the time step size corresponds to the time in which the runner rotates at $\Delta \varphi=1^{0}$. The results of the modelling are stored at every time step.

\section{Results}

Two runners with a different pitch angle $\left(\varphi_{\mathrm{r}}\right)$ have been calculated. The obtained results give us an opportunity to examine the dependency $c_{p}=f(T S R)$. To verify the accuracy of the calculations, the results of the physical experiments are imposed on the graphs (fig. 4), both in a dimensionless form.

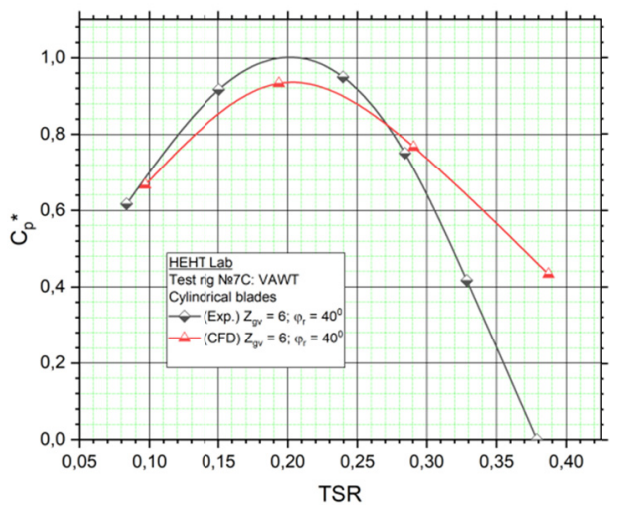

a.

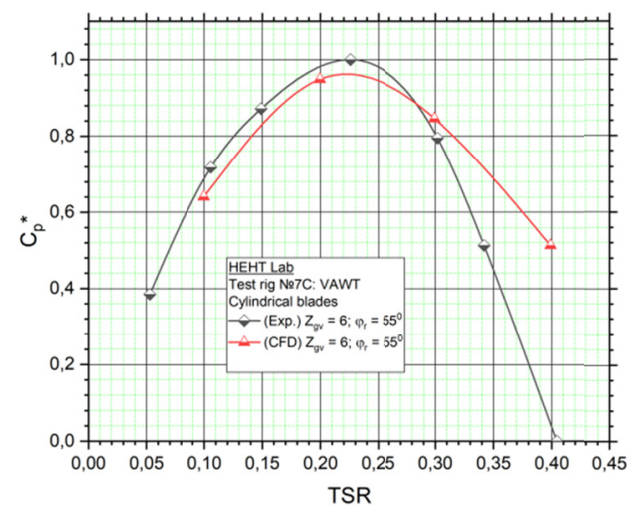

b.

Fig. 4. Performance characteristics of a runner with 6 blades at $40^{\circ}$ (a) and $55^{\circ}$ (b) pitch angles.

\section{Analysis}

The analysis of the results of the experimental studies leads to the following important conclusions:

- The law of change of the theoretical characteristics is similar to that of the real ones. 
- Numerical results predict the location of the maximum value of the power coefficient with an error up to $0.3 \%$.

- The differences in the maximum value of the power coefficient between the numerical and experiment data is $7 \%$ (Fig. $4 \mathrm{a}$ ) and $4 \%$ (Fig. $4 \mathrm{~b}$ ).

- Numerical data shows larger values of a power coefficient at values of tip speed ratio larger than 0.27 (Fig. 4a) and 0.285 (Fig. 4b). The maximum difference is $41.7 \%$ (Fig. 4a) and $51.3 \%$ (Fig.4 b).

- Numerical and experimental data give similar results at values of tip speed ratio lower than 0.27 (Fig. 4a) and 0.285 (Fig. 4b).

The numerical and the experimental data from other similar studies $[6,7]$ shows up to $57 \%$ difference of power coefficient with k-omega SST turbulence model and $20 \%$ with RNG $\mathrm{k}-\varepsilon$ turbulence model.

\section{Conclusion}

The main results of this study are expressed as following:

1. A numerical study of a model Darrieus VAWT has been made.

2. Two runners have been examined, with a different pitch angle of the blades.

3. The obtained results have been validated on test rig №7C in HEHT Lab [3, 4].

4. The graphs show that the turbulence k-omega model can predict the performance characteristics of Darrieus VAWT with up to 7\% error in the optimal operating regime.

\section{References}

1. N. Batista, R. Melício, V. Mendes, J. Figueriedo, A. Reis. Darrieus Wind Turbine Performance Prediction: Computational Modeling. IFIP, series IFIPAICT, vol, 394, pp. 382-391, (2013).

2. Parachivoiu, I. Wind Turbine Design: with emphasis on Darrieus conscept, 1 st edn. Polytehnic International Press, Canada (2002).

3. Obretenov, V., R. Iliev. A new model vertical axis wind turbine. Proceedings of the Scientific Conference EMF`2018, vol. II, pp. 288-295, Sozopol, (2018), in Bulgarian.

4. Obretenov, V., Ts.Tsalov. Guide for laboratory exercises in hydro and wind energy. (machinery and equipment). 'Publishing house of the University of Ruse', (2017), in Bulgarian.

5. Obretenov, V., Ts. Tsalov, Ch. Chakarov. Vertical axis wind turbine. Proceedings of the Scientific Conference EMF '2012, vol. II, pp. 28-34, Sozopol (2012), in Bulgarian.

6. Almohammadi, K.M., D.B. Ingham, L. Ma, M. Pourkashan. Computational fluid dynamics (CFD) mesh independency techniques. Energy vol. 58, pp. 483-493, (2013).

7. Balduzzi, F., A. Bianchini, R. Maleci, G. Ferrara, L. Ferrari. Critical issues in the CFD simulation of Darrieus wind turbines. Renew Energy vol. 85, pp. 419-435, (2016).

8. Ahmedov, A, K Tujarov, G. Popov. Methodology for Numerical Modeling the Performance of Vertical Axis Wind Turbines. Proceedings of the University of Ruse, vol 53, pp. 194 - 200, Ruse, (2014), in Bulgarian.

9. Obretenov, V.S., Ts. Tsalov. The new hydraulic laboratory at the Technical University of Sofia. Proceedings of the IX international scientific and technical conference 'Hydraulic machines, hydraulic drives and hydro- and pneumatic automation. Current status and development prospects' pp. 79-87, St. Petersburg, (2016). 